\title{
SISTEMÁTICA DOS CONULÁRIOS (CNIDARIA) DA FORMAÇÃO PONTA GROSSA (DEVONIANO), ESTADO DO PARANÁ, BACIA DO PARANÁ, BRASIL
}

\author{
JULIANA DE MORAES LEME, SABRINA COELHO RODRIGUES \\ Depto. de Geologia Sedimentar e Ambiental, IG, USP, Rua do Lago, 562, 05508-900, São Paulo, SP, Brasil. \\ leme@usp.br,scoelho@usp.br \\ MARCELLO GUIMARÃES SIMÕES \\ Depto. de Zoologia, IB, UNESP, Campus Rubião Júnior, 18618-000, Cx. P. 510, Botucatu, SP, Brasil. \\ btsimoes@ibb.unesp.br \\ HEYO VAN ITEN \\ Department of Geology, Hanover College, 47243, Hanover, IN, USA. vaniten@hanover.edu
}

\begin{abstract}
RESUMO - Revisa-se aqui a fauna de conulários (Cnidaria) fósseis presente na Formação Ponta Grossa (Devoniano, sub-bacia Apucarana, Estado do Paraná, municípios de Jaguariaíva e Ponta Grossa), originalmente descrita em 1913 e que revelou, na época, a presença de três espécies, Conularia quichua Ulrich (in Steinmann \& Doderlein, 1890), Paraconularia africana (Sharpe, 1856) e P. ulrichana (Clarke, 1913). O exame de 133 espécimes, provenientes de rochas litoestratigraficamente correspondentes ao Membro Jaguariaíva (Sequiência B), revela a ocorrência das espécies Conularia quichua e Paraconularia africana na Formação Ponta Grossa; $P$. ulrichana (Clarke, 1913), contudo, não foi encontrada no material estudado. C. quichua é a espécie mais abundante e amplamente distribuída na seção geológica de Jaguariaíva, sendo rara a ocorrência de $P$. africana. Os novos dados permitem concluir que a fauna de conulários da Formação Ponta Grossa é pouco diversificada, especialmente se comparada com faunas coevas do Domínio Malvinocáfrico. Notavelmente, os espécimes de C. quichua e $P$. africana estudados apresentam os caracteres morfológicos internos da teca preservados e aqui descritos pela primeira vez. Conseqüentemente, as diagnoses específicas de $C$. quichua e $P$. africana foram emendadas.
\end{abstract}

Palavras-chave: Conulatae, sistemática, Devoniano, Formação Ponta Grossa, bacia do Paraná.

ABSTRACT - SYSTEMATICS OF CONULARIIDS (CNIDARIA) OF THE PONTA GROSSA FORMATION (DEVONIAN), PARANÁ STATE, PARANÁ BASIN, BRAZIL. The conulariid (Cnidaria) fossils of the Ponta Grossa Formation (Devonian, Apucarana sub-basin, Paraná State, Jaguariaíva and Ponta Grossa Counties), originally described in 1913 and composed of Conularia quichua Ulrich (in Steinmann \& Doderlein, 1890), Paraconularia africana (Sharpe, 1856) and P. ulrichana (Clarke, 1913) is revised here. The exam of 133 specimens from rocks that are lithostratigraphically coeval to the Jaguariaíva Member (Sequence B) indicates the presence of Conularia quichua and Paraconularia africana in the Ponta Grossa Formation, but P. ulrichana was not found in the studied material. $C$. quichua is the commonest and widespread species in the geological section at Jaguariaíva, while $P$. africana is rare. The new data allows to conclude that the conulariid fauna of the Ponta Grossa Formation is poorly diversified, especially if compared with other coeval faunas of the Malvinokaffric Realm. Notably some internal characters are preserved in thecae of $C$. quichua and $P$. africana. Such features are here described by the first time. Consequently, the specific diagnosis of $C$. quichua and $P$. africana were emended.

Key words: Conulatae, systematic, Devonian, Ponta Grossa Formation, Paraná basin.

\section{INTRODUÇÃO}

Conulários (Conulatae, sensu Collins et al., 2000) são cnidários, extintos, bentônicos, sésseis de epifauna, com teca fosfática, piramidal, alongada e, na maioria das vezes, com quatro faces (Babcock, 1991; Van Iten, 1991a; Leme, 2002). Representantes desse enigmático grupo de invertebrados ocorrem em uma ampla variedade de fácies sedimentares, especialmente de águas rasas, plataformais, em ambientes siliciclásticos e carbonáticos do Neocambriano ao Triássico (McMenamin, 1987; Jerre, 1994; Van Iten, 1991b; Van Iten et al., 1996), embora Ivantsov \& Fedonkin (2002), sugiram possíveis ocorrências de formas aparentadas no Neoproterozóico.

Conulários são característicos das faunas do Domínio Malvinocáfrico (sensu Richter \& Richter, 1942, e.g., Clarke, 
1913; Kozlowski, 1913, 1923; Babcock et al., 1987a), sendo extremamente abundantes e diversificados nas concentrações fossilíferas do Devoniano da Bolivía (e.g., Babcock et al., 1987a). Outras ocorrências importantes na América do Sul são verificadas também na Argentina (e.g., Thomas, 1905), no Peru (e.g., Steinmann, 1930), no Paraguai (Babcock et al., 1990), no Uruguai (e.g., Mendéz-Alzola \& Sprechmann, 1973) e na África do Sul (e.g., Schwarz, 1906). Na bacia do Paraná, ocorrências espetaculares de conulários (dada sua abundân-
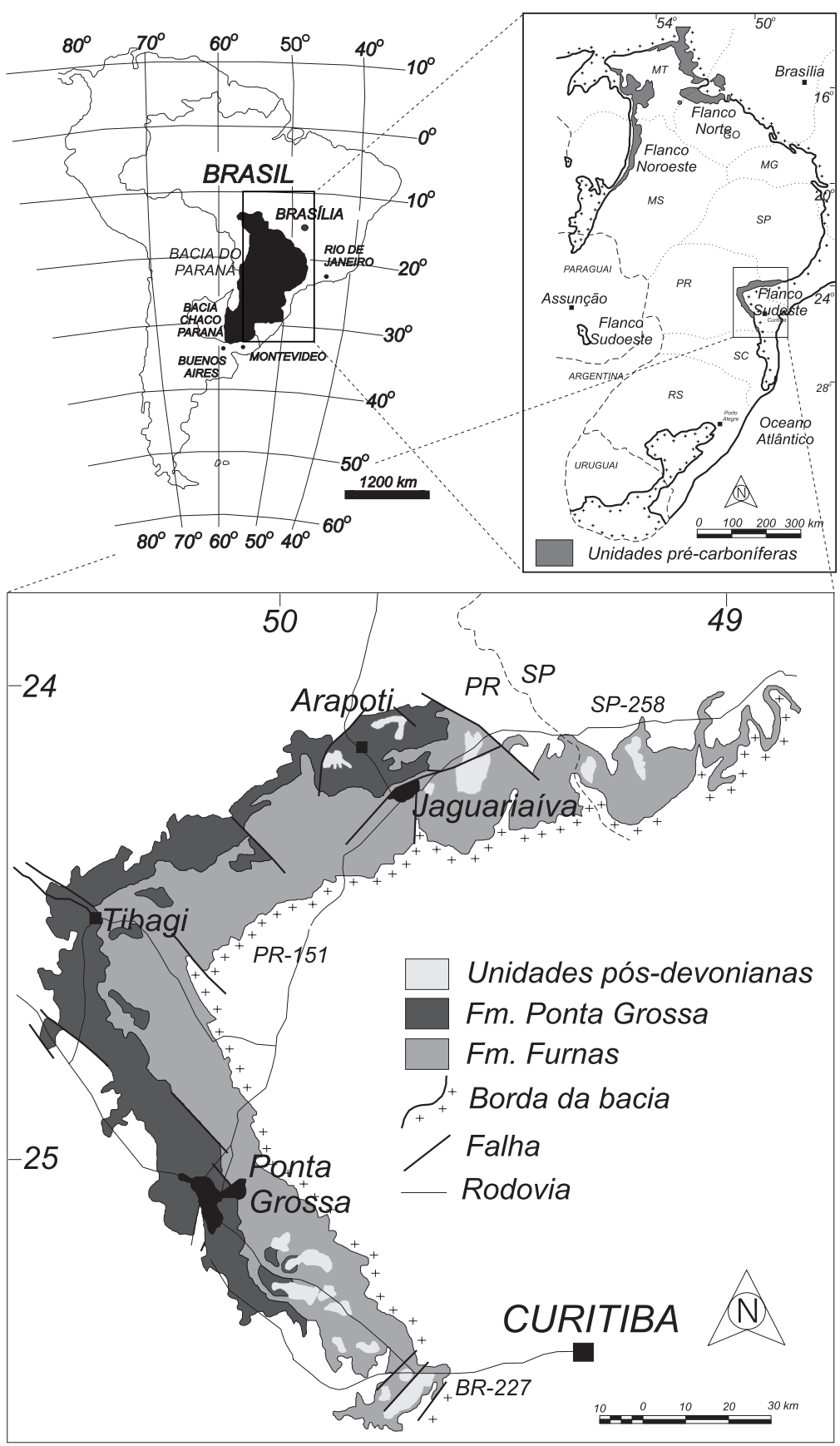

Figura 1. Mapa de localização dos afloramentos da Formação Ponta Grossa, mostrando a faixa de afloramentos na borda leste da Bacia do Paraná.

Figure 1. Location map of studied outcrops of the Ponta Grossa Formation, also showing the Pre-Carboniferous outcrop belt in the eastern border of the Paraná Basin. cia, qualidade e modo de preservação) são verificadas na Formação Ponta Grossa (Pragiano-Emsiano, Grahn et al., 2000 - vide Bergamaschi, 1999 e Bergamaschi \& Pereira, 2001, para uma interpretação diferente da idade da Formação Ponta Grossa) (Clarke, 1913; Kozlowski, 1913; Simões et al, 2000b).

Contrariamente a outros grupos de invertebrados marinhos, comuns na Formação Ponta Grossa, tais como trilobitas (e.g., Popp, 1985), braquiópodes (e.g., Quadros, 1987; Bosetti, 1989), moluscos bivalves (e.g., Kotzian, 1995; Machado, 1999) e tentaculites (e.g., Ciguel, 1989; Azevedo-Soares, 1999) que, nas últimas décadas, foram alvo de revisões importantes, os conulários permaneceram praticamente ignorados (ver menções em Lange \& Petri, 1967; Petri, 1948; Petri \& Fúlfaro, 1983) e inadequadamente descritos, face ao enorme avanço ocorrido na sistemática e no conhecimento morfológico do grupo, particularmente a partir de meados da década de 1980.

Curiosamente, nos últimos cinco anos, graças aos esforços de pesquisadores da UNESP, UFRJ, Museu Nacional e do apoio da FAPESP e do Programa de Pós-graduação em Geologia Sedimentar do IG/USP, houve um renovado interesse no estudo dos conulários da bacia do Paraná. Os resultados dessas pesquisas aparecem, entretanto, em documentos de pequena circulação, tais como as dissertações de mestrado de Leme (2002), de Rodrigues (2002) e de Siviero (2002). Essas pesquisas são importantes porque, a despeito da qualidade das descrições e ilustrações de Clarke (1913), esse estudo não está, obviamente, atualizado. De fato, desde Clarke (1913), nenhum estudo taxonômico formal foi publicado. Nesse contexto, as descrições de Clarke (1913) carecem de revisão, face ao avanço no estudo dos conulários (e.g., Babcock \& Feldmann, 1986; Jerre, 1993; 1994; Van Iten, 1991a,b; Van Iten, 1992a,b; Van Iten et al., 1996; Simões et al., 2000b). Em adição, é importante lembrar que, em muitos casos, não é possível resgatar determinadas feições morfológicas da teca (e.g., morfologia de nodos e cristas), apenas via as ilustrações de Clarke (1913). Além disso, algumas espécies da fauna da Formação Ponta Grossa, tais como C. quichua, não foram sequer ilustradas, dada a sua péssima qualidade de preservação (Clarke, 1913, p. 163). Conforme será visto mais adiante, muitas das feições que hoje são utilizadas na descrição e na identificação dos conulários, são feições internas à teca (Van Iten, 1992b). Tais feições nunca foram descritas por Clarke (1913), sendo apresentadas aqui pela primeira vez. Isso só foi possível em decorrência do grande número de exemplares examinados e da qualidade de preservação do material investigado. Dessa forma, o presente estudo objetiva a revisão sistemática dos conulários 
(Cnidaria) da Formação Ponta Grossa, na sub-bacia Apucarana, a partir do minucioso estudo morfológico de tecas provenientes dos municípios de Jaguariaíva e Ponta Grossa, Estado do Paraná.

\section{MATERIALE MÉTODOS}

Material examinado, procedência e posição estratigráfica

Os espécimes aqui descritos provêm de sedimentitos litoestratigraficamente correspondentes ao Membro Jaguariaíva (Lange \& Petri, 1967) ou à Sequiência B de Bergamaschi (1999) e de Bergamaschi \& Pereira (2001) da Formação Ponta Grossa, nos municípios de Jaguariaíva e Ponta Grossa, no Paraná (Figura 1). Em sua grande maioria, os espécimes foram coletados nos afloramentos situados nos quilômetros 3,8 e 4,5 do corte da estrada de ferro Jaguariaíva-Arapoti ou, respectivamente, a 29 e $44 \mathrm{~m}$ da base da Formação Ponta Grossa na área de estudo. Apenas uma ocorrência isolada foi registrada a $39 \mathrm{~m}$ da base (Figura 2). Nos intervalos acima, isso é, a 29 e $44 \mathrm{~m}$ da base, predominam os horizontes representados por depósitos de sufocamento (Simões et al., 2000a,b), que são progressivamente sucedidos por folhelho cinza escuro laminado e siltitos e arenitos interlaminados, intensamente bioturbados (Bergamaschi \& Pereira, 2001) (Figura 2). Segundo esses autores, tais folhelhos representam superfícies de inundação marinhas.

Já a posição estratigráfica dos espécimes provenientes do município de Ponta Grossa é incerta. Tais espécimes estavam depositados na coleção da UEPG e, segundo Bosetti (informação pessoal, 2001), foram provavelmente coletados nos afloramentos informalmente designados por Kotzian (1995) de Curva I e Curva II. Os sedimentitos aflorantes nessa seção são, contudo, similares àqueles de Jaguariaíva, tanto em seu conteúdo fossilífero, como nas estruturas sedimentares.

Os espécimes estudados apresentam-se em geral incompletos, com a região basal parcialmente preservada. Os fósseis mantêm ainda uma fina película carbonosa recobrindo externamente a teca. Essa, entretanto, pode mostrar diferentes estágios de esfoliação, dependendo de sua exposição ao intemperismo (Rodrigues, 2002). Reconhecer tal feição é importante, porque é na película carbonosa que estão preservadas as principais características morfológicas empregadas na descrição e na classificação dos conulários.

Em adição ao material acima, foram examinados exemplares de conulários depositados nas coleções científicas da Universidade Estadual de Ponta Grossa (UEPG), da Universidade de Guarulhos (UNG) e da Universidade de São Paulo (USP). Assim, no total, foram preparados 133 exemplares de conulários, dos quais 126 provenientes de Jaguariaíva e 7 de Ponta Grossa. Esse material está assim distribuído nas coleções examinadas: 116 exemplares na Coleção Científica do Departamento de Zoologia do IBB/UNESP (DZP), 6 na Coleção Científica do IGc/USP (GP/E), 10 na Coleção Científica da UNG (CuPg) e 1 na Coleção Científica da UEPG (UEPG). O material foi preparado segundo as técnicas usuais em estudos de macroinvertebrados fósseis (Feldmann et al., 1989).

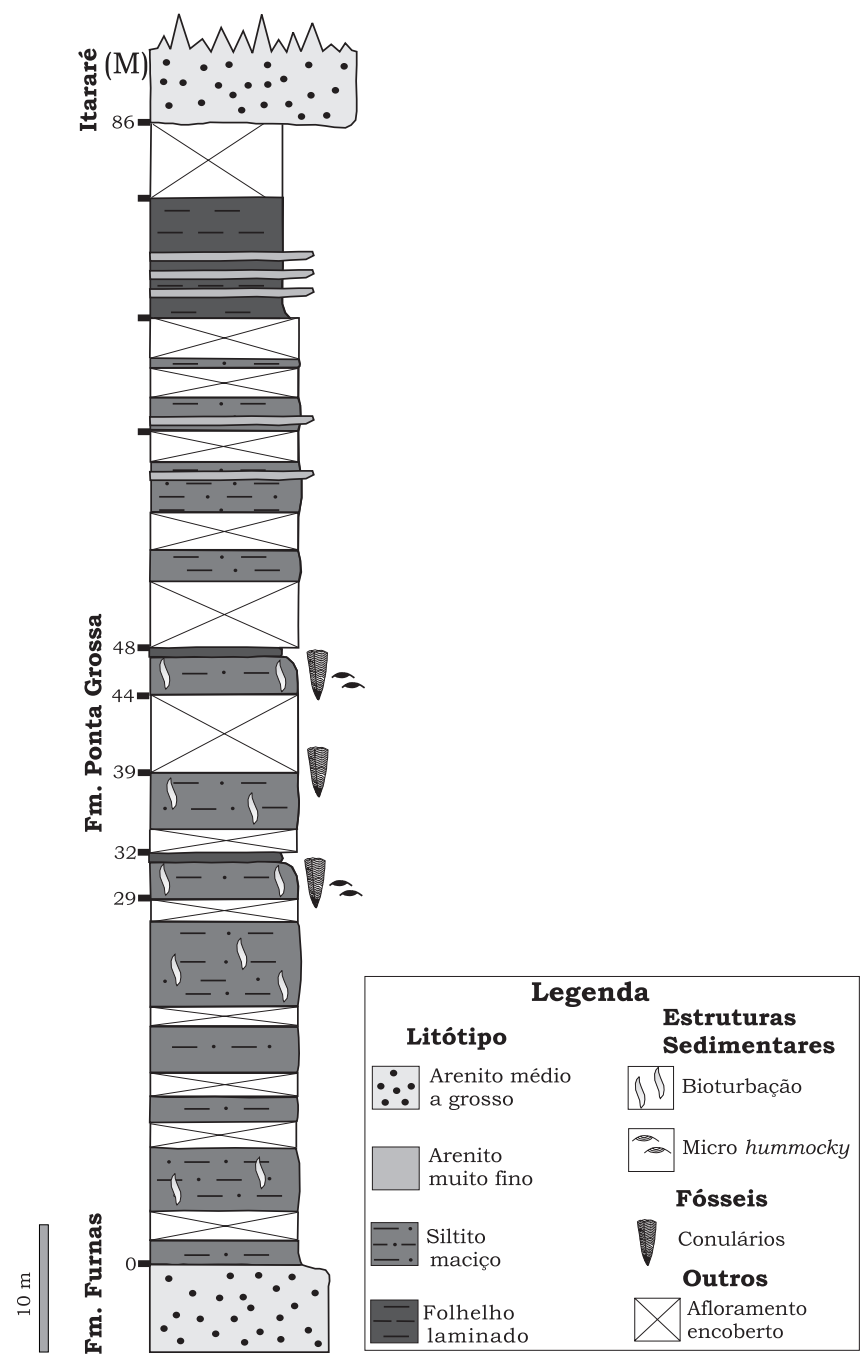

Figura 2. Seção colunar do afloramento situado na estrada de ferro Jaguariaíva-Arapoti (PR), Formação Ponta Grossa, Seqüência B (modificado de Bergamaschi, 1999). Observar a distribuição vertical dos conulários.

Figure 2. Columnar section of the outcrop located at JaguariaívaArapoti railroad, Paraná State, Ponta Grossa Formation, Sequence B (modified from Bergamaschi, 1999). Note the vertical distribution of conulariids.

A revisão da literatura mostra que diversos autores têm utilizado diferentes atributos e termos para a descrição da morfologia da teca dos conulários (Figura 3). Bischoff (1978) utilizou as características internas da teca para a descrição de conulários do Eossiluriano da Austrália, enquanto Sinclair (1940, 1942, 1948, 1952), Van Iten (1991a,b) e Van Iten et al. (1996) empregaram uma combinação de feições externas e internas na identificação de espécimes da América do Norte. Uma leitura atenta desses trabalhos mostra, entretanto, que os termos empregados são imprecisos, com múltiplos significados. Em adição, não há ainda um entendimento adequado dos aspectos ontogenéticos, evolutivos e funcionais da morfologia da teca dos conulários (Bishoff, 1978). Conseqüentemente, isso dificulta o estabelecimento de homologias, fazendo com que o estudo da morfologia dos conulários seja controverso. 


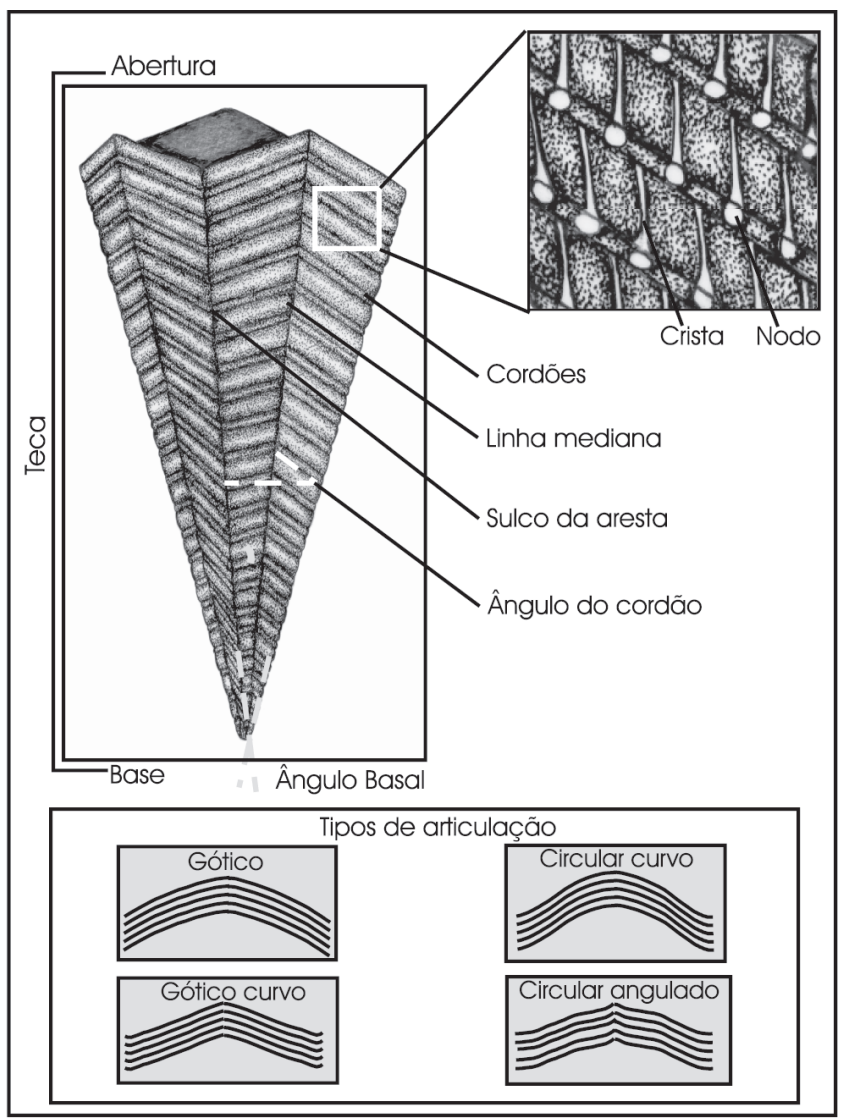

Figura 3. Morfologia geral dos conulários, com as principais feições e termos morfológicos utilizados nas descrições anatômicas.

Figure 3. Conulariid general morphology, with the main anatomical terms applied.

Uma tentativa de padronização da terminologia morfológica da teca dos conulários foi apresentada por Babcock \& Feldmann (1986). Esses autores selecionaram e definiram, principalmente, feições morfológicas externas, muitas passíveis de quantificação, tais como, espaçamento relativo dos cordões, ângulo basal, espaçamento entre nodos e cristas e ângulo dos cordões. Com exceção do padrão de articulação dos cordões e da presença ou ausência de nodos e cristas, nenhum outro caráter qualitativo foi utilizado na diagnose de gêneros e espécies. De acordo com esses autores, algumas feições biométricas poderiam ser empregadas para definir gêneros (e.g., espaçamento relativo dos cordões) e espécies (e.g., ângulo do cordão). Embora na prática taxonômica algumas dessas feições tenham sido amplamente utilizadas desde Sinclair (1940), notou-se que muitos dos caracteres morfométricos (e.g., ângulo basal, ângulo do cordão, espaçamento entre cordões) variam de acordo com o modo de preservação, o que coloca em cheque a sua validade (Leme, 2002; Rodrigues, 2002). Desse modo, seguiram-se aqui as recomendações sugeridas por Rodrigues (2002) e Leme (2002), a saber: (i) fundamentou-se o estudo em uma coleção numerosa, para identificar o maior espectro possível de variações morfológicas, decorrentes de alterações tafonômicas; (ii) priorizou-se o exame de espécimes pouco deformados (bem preservados); e (iii) as descrições fundamentaram-se em exem- plares completos, ou em grande parte completos, pois muitas feições (e.g., padrão de articulação) variam ao longo da teca. Além disso, as feições biométricas, tais como o ângulo basal e o ângulo do cordão, foram excluídas da descrição porque são passíveis de alterações durante a fossilização (Leme, 2002; Rodrigues, 2002). Entretanto, a feição espaçamento entre os cordões foi utilizada, seguindo as recomendações e cuidados já mencionados.

Quanto aos termos morfológicos, utilizou-se uma combinação abrangendo os termos sugeridos por Sinclair (1940, 1942, 1948, 1952), Moore \& Harrington (1956a,b), Bischoff (1978), Van Iten (1991a,b) e Van Iten et al. (1996). Adicionalmente, os termos empregados na descrição da articulação dos cordões seguem Babcock \& Feldmann (1986).

\section{SISTEMÁTICA PALEONTOLÓGICA
Subclasse CONULATAE Collins et al., 2000
Família CONULARIIDAE Walcott, 1886
Gênero Conularia Sowerby, 1820

Diagnose emendada. Conulários com cordões opostos na região da linha mediana, geralmente contínuos no sulco da aresta, onde se curvam levemente em direção à abertura; articulação do tipo gótico ou circular angulado; interespaços geralmente atravessados por cristas que não se alinham longitudinalmente; nodos presentes. Espessamentos internos na aresta (Figura 4.7-4.9 e 4.11).

\section{Conularia quichua Ulrich, 1890 (Figura 4)}

Conularia quichua Ulrich in Steinmann \& Döderlein, 1890: 343, fig. 395d-e; Ulrich, 1892: 34-36, pl. 3, fig. 7a-b; Bernard, 1895, fig. 265c; Reed, 1904: 248, pl. 30, figs. 10-10a; Clarke, 1913: 163; Douglas, 1920: 37-38, pl. 1, fig. 3; Kozlowski, 1923: 69-70, pl. 7, fig. 6; Reed, 1925: 107; Ulrich in Steinmann, 1930, figs. 21d-e; Kiderlen, 1937, fig. 10; Ahlfeld \& Branisa, 1960, pl. 4, figs. 4, 10-10a; Hasman et al., 1962: 21; Branisa, 1965, pl. 43, figs. 1, 3-4; pl. 45, figs. 2-4, 6-12; Babcock et al., 1987a: 218, fig. 4; Leme, 2002: 33, pl. 1, 2.

Conularia quichua Ulrich? Kayser, 1897: 288, pl. 11, figs. 12; Thomas, 1905: 254-255, pl. 12, fig. 19.

Conularia (Mesoconularia) quichua Ulrich. Boucek, 1939, fig. 3e.

Mesoconularia quichua Ulrich. Sinclair, 1948:119.

Conularia cf. undulata Conrad. Sensu Reed, 1904: 248-249, pl. 31, figs. 1-1a; Hansman et al., 1962: 21-22.

Conularia cf. quichua Ulrich. Ahlfeld \& Branisa, 1960, pl. 4, fig. 9; Lof, 1985.

Conularia quichua var. nov. Branisa, 1965, pl. 43, fig. 2.

Conularia sp. Branisa, 1965, pl. 43, figs. 5-6.

Diagnose emendada. Conulários com articulação dos cordões do tipo circular angulado nas regiões da abertura e mediana (Figura 4.1 e 4.2), e gótico a $10 \mathrm{~mm}$ da base (Figura 4.3); cristas longas e afiladas, atravessando todo o comprimento dos 

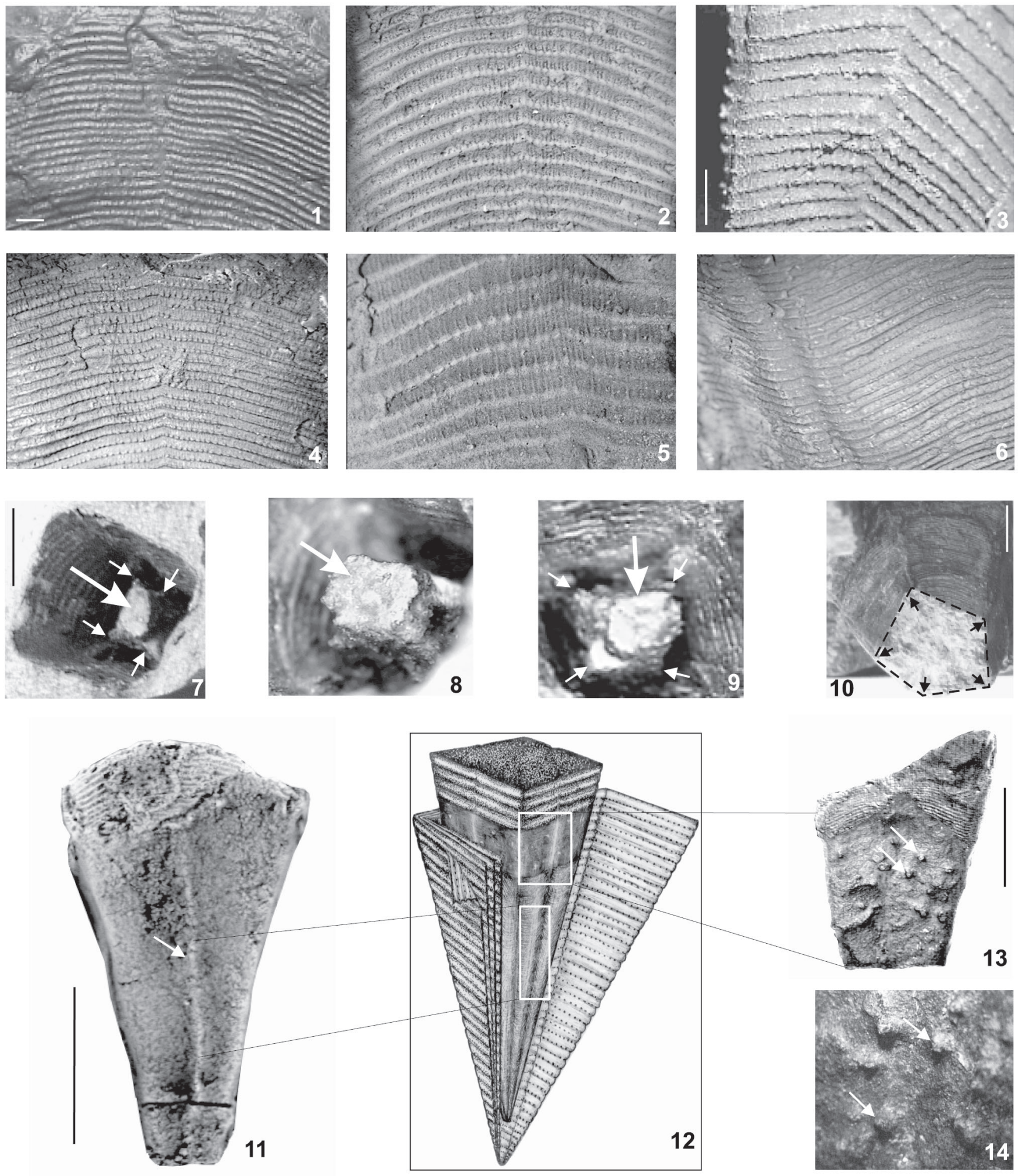

Figura 4. Conularia quichua. 1-3. Articulação dos cordões: 1. (DZP-3082) região adapertural; 2. (DZP-3515) região mediana x0,8; 3 , (GP/ 1E-4914) região basal. 4-5. (DZP-3509) Nodos e cristas $(4, x 0,6 ; 5, x 1,2)$. 6. (DZP-3509) Cordões no sulco da aresta (x0,6). 7-9. (DZP3073) Pilar axial e carenas na base $(8, x 1,2 ; 9, x 1,2)$. 10. (DZP-3081) 5 faces. 11. (DZP-3516) Linha mediana espessada. 12. Esquema de conulário. 13-14. (DZP-3082) Pontuações indeterminadas na abertura (13, detalhe das pontuações, $x 1,6)$. Setas em branco: carenas, septo e pontuações; setas em preto: arestas; escala gráfica: 1 e $3(1 \mathrm{~mm}) ; 7,11$ e $14(1 \mathrm{~cm})$.

Figure 4. Conularia quichua. 1-3. Rods articulation: 1. (DZP-3082) adapertural region; 2. (DZP-3515) median region, x0,8; 3. (GP/1E-4914) basal region. 4-5. (DZP-3509) Nodes and crests (4, x0,6; 5, x1,2). 6. (DZP-3509) Rods in the corner grooves (x0,6). 7-9. (DZP-3073) Axial pilar and carinae at the base $(8, x 1,2 ; 9, x 1,2) .10$. (DZP-3081) 5 faces. 11. (DZP-3516) Thickened midline. 12. Conulariid sketch. 13-14. (DZP-3082) Undetermined internal pits (adapertural region; 13, pits in details, x1,6). White arrows: carinae, septa and pits; black arrows: corners; graphic scale: 1 and $3(1 \mathrm{~mm}) ; 7,11$ and $14(1 \mathrm{~cm})$. 
interespaços (Figura 4.5). Arestas espessadas na região basal, estendendo-se a um pilar axial aparentemente de mesma constituição da teca (Figura 4.7-4.9); linha mediana com espessamentos internos (Figura 4.11) e pontuações irregularmente distribuídas na região da abertura da teca (Figura 4.13 e 4.14).

Descrição. Conulário completo, faltando a base em alguns exemplares $(\mathrm{n}=47)$, apresentando quatro ou raramente cinco faces (Figura 4.10) e comprimento variando de 5,94 a 9,55 cm. Faces semelhantes na largura. Carenas presentes nas quatro arestas e restritas à região basal (Figura 4.7-4.9). Estrutura a moda de um pilar axial (Figura 4.7-4.9), apresentando mesma constituição da teca, conectada às carenas. Presença de espessamentos internos na região da linha mediana (Figura 4.11). Pontuações de natureza não conhecida, irregularmente distribuídas na região da abertura da teca (Figura 4.13 e 4.14). Cordões sempre opostos (contínuos) na linha mediana (Figura 4.1-4.5), contínuos no sulco da aresta (Figura 4.6), em número de 30 a 40 por centímetro, apresentando articulação do tipo circular angulado nas regiões da abertura e mediana (Figura 4.1 e 4.2) e gótico a $10 \mathrm{~mm}$ da base (Figura 4.3). Nodos pequenos e hemisféricos (Figura 4.4 e 4.5 ), em número de 4 a
5 por milímetro, concentrados ( 4 a $7 / \mathrm{mm})$ nas proximidades das arestas e linhas medianas. Cristas adaperturais longas e afiladas, atravessando todo o comprimento dos interespaços, terminando, predominantemente, entre dois nodos (Figura 4.5). Crista adapical ausente. Parede basal presente no espécime DZP-3611a.

Ocorrências e horizontes fossilíferos. Formação Ponta Grossa (Pragiano-Emsiano), Membro Jaguariaíva (Seqüência B). Afloramento situado nos quilômetros 3,8 e 4,5 da estrada de ferro (Ramal Jaguariaíva-Arapoti, Jaguariaíva, PR), nos intervalos entre 29-32 m, a 39 m e entre 44-48 m, a partir do contato basal com a Formação Furnas. Afloramentos "Curva I" e "Curva II" (Ponta Grossa, PR).

Material tipo. Holótipo perdido (Babcock et al., 1987a). YPFB 3432 e USNM 409818 (neótipos).

Material examinado. Espécimes IBB/UNESP, DZP-3073-3085, 3316-3318, 3321-3324, 3327, 3329-3331, 3502-3505, 3507-3517, 3609-3621, 3623-3624, 3626-3634, 3684, 3686, 16814-16815, 16818-16820, 17036-17053, 17057-17058; UEPG-D-058; GSA/ IG-USP, GP/1E-2575, 3177-3178,3191,4914, GP/3E-3569; UNGCuPg2, 4, 9, 10, 14.
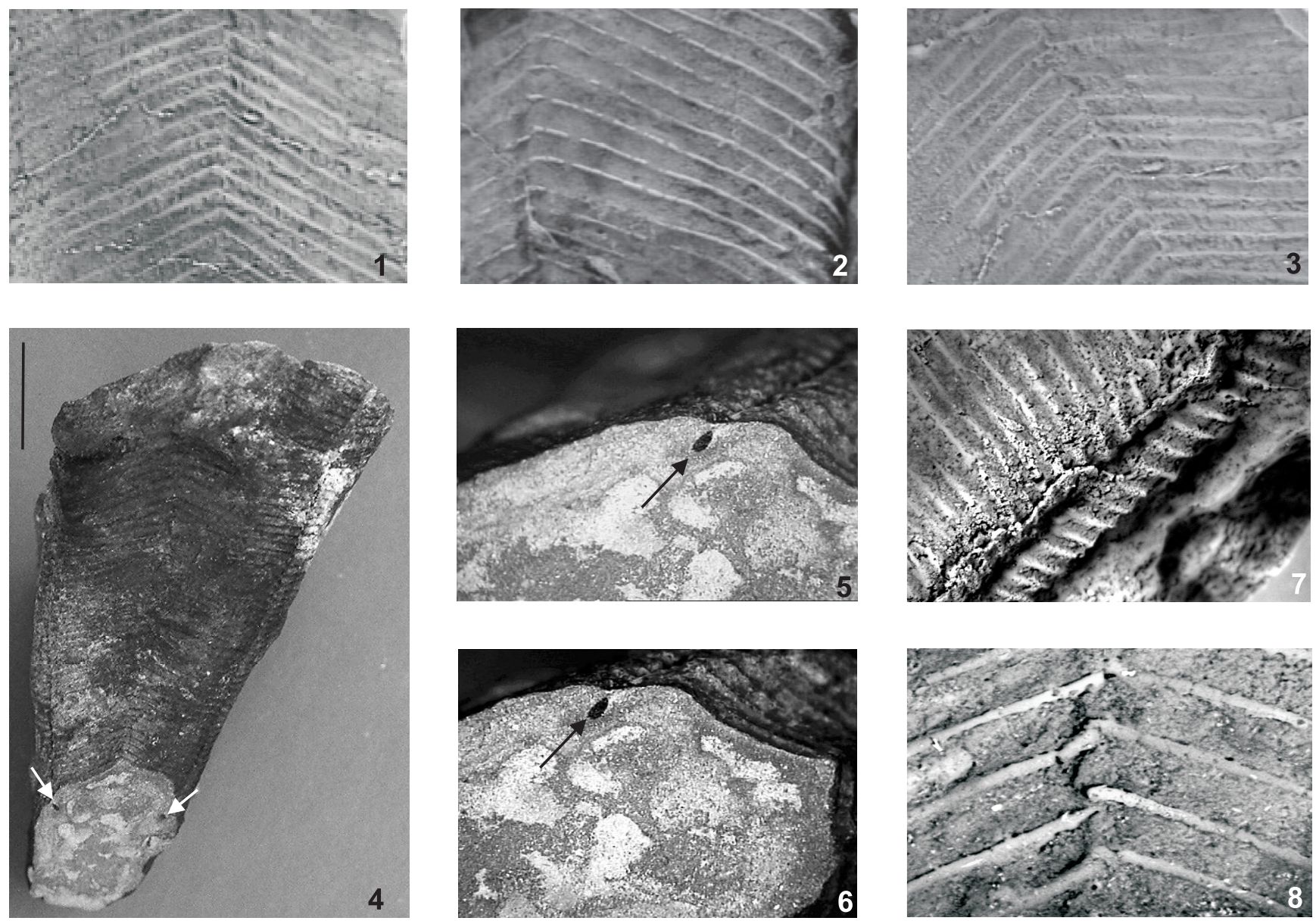

Figura 5. Paraconularia africana. 1-3. Articulação dos cordões (CuPg-01): 1. região adapertural; 2. região mediana; 3. região basal. 4-6. Carenas (GP/3E-4194): 5-6. Detalhe das carenas (setas) nas arestas (x0,8). 7. Cordões no sulco da aresta (CuPg-01, x0,6). 8. Encontro dos cordões na região da linha mediana $(x 0,8)$. Escala $=1 \mathrm{~cm}$.

Figure 5. Paraconularia africana. 1-3. Rods articulation (CuPg-01): 1. adapertural region; 2. median region; 3. basal region. 4-6. Carinae (GP/3E-4194): 5-6. Carinae (arrows) details in the corner grooves (x0,8); 7. Rods in the corner grooves $($ CuPg-01, x0,6); 8 . Rods in the midlines $(\times 0,8)$. Scale bar: $1 \mathrm{~cm}$. 
Discussão. Segundo Babcock et al. (1987a), C. quichua é muito semelhante a $C$. niagarensis por possuir articulação dos cordões do tipo circular angulado, na maior parte da teca e a única diferença entre $C$. quichua e $C$. niagarensis seria o ângulo basal. Porém, $C$. quichua apresenta articulação dos cordões do tipo gótico na região basal e as diferenças na preservação entre espécimes inflados e fortemente achatados não permitem o uso do ângulo basal na diagnose específica.

Outra diferença marcante entre $C$. quichua e as demais espécies de Conularia é a ocorrência de espécimes mostrando cinco faces verdadeiras (Figura 4.10). A ocorrência de tecas não apresentando quatro faces tem sido observada em outros gêneros de conulários, incluindo Conularina triangulata (Raymond) (três faces) e Paraconularia missouriensis (Swallow) (seis faces) (Babcock et al., 1987b), mas não em Conularia. Portanto, o espécime de C. quichua (DZP-3081b, Figura 4.10) com teca exibindo cinco faces é o primeiro do gênero com essa condição a ser documentado na literatura.

Finalmente, as feições internas tais como, arestas espessadas na região basal, pilar axial, linha mediana espessada e pontuações irregularmente distribuídas na região da abertura da teca, não foram referidas para nenhum exemplar dessa espécie, mesmo os pertencentes à numerosa coleção da Bolívia (Babcock et al., 1987a).

Gênero Paraconularia Sinclair, 1940

Paraconularia africana (Sharpe, 1856) (Figura 5)

Conularia africana Sharpe, 1856, p. 214, Est. 27, figs. 13a-b; Ulrich, 1892, p. 29-31, Est. 3, fig. 4; Reed, 1904, p. 247-248; Schwarz, 1906, p. 362-363, Est. 7, figs. 13, 15; Reed, 1925, p. 103, Kiderlen, 1937, figs. 15, 26; 1-2; Moore \& Harrington, 1956b, p. F60-F61, figs. 46, 11; Ahlfeld \& Branisa, 1960, Est. 4, fig. 13; Hansman et al., 1962, p. 21; Branisa, 1965, Est. 44, figs. 1-3, 6, 12, 14-16; Méndez-Alzola \& Sprechmann, 1973, p. 130, Est. 1, fig. 1; Babcock et al., 1987a, figs. 4a-d, 8c, Tab. 1. Conularia africana var. striatula Kozlowski, 1923, p. 67-68, Est. 7, figs. 1-3.

Paraconularia africana (Sharpe). Sinclair, 1948, p. 187-188; Babcock et al., 1987a, p. 221, fig. 7; Leme, 2002, p. 42 , Est. 3.

Conularia s.1. striatula Kozlowski. Sinclair, 1948, p. 285. Conularia africana striatula Kozlowki. Ahlfeld \& Branisa, 1960, Est. 4, fig. 3; Branisa, 1965, Est. 44, figs. 5, 7, 10.

Conularia sp. Branisa, 1965, Est. 44, fig. 4, Est. 78, fig. 5. Conularia baini Ulrich. Sensu Schwarz, 1906, p. 362, Est. 8 fig. 14; Branisa, 1965, Est. 44, figs. 14-16.

Diagnose emendada. Conulários que possuem articulação dos cordões do tipo gótico ao longo da teca (Figura 5.1-5.3); espaçamento entre os cordões, variando em número de 10 a 30 cordões por centímetro; nodos e cristas adaperturais presentes. Arestas internamente espessadas (carenadas) (Figura 5.4-5.6).
Descrição. Conulário completo, apresentando quatro faces e comprimento máximo de $12,2 \mathrm{~cm}$. Carenas presentes ao longo das arestas (Figura 5.4-5.6). Espessamentos internos na região da linha mediana ausentes. Cordões alternados na linha mediana (Figura 5.8), descontínuos no sulco da aresta (Figura 5.7), em número de 10 a 30 por centímetro, apresentando articulação do tipo gótico ao longo da teca (Figura 5.15.3). Nodos, cristas e parede basal ausentes.

Ocorrências e horizonte fossilífero. Formação Ponta Grossa (Pragiano-Emsiano), Membro Jaguariaíva (Seqüência B). Afloramento situado no km 4,5 da estrada de ferro (Ramal Jaguariaíva-Jacarezinho, Jaguariaíva, PR), no intervalo entre 44-48 m a partir do contato basal com a Formação Furnas.

Material tipo. Holótipo perdido (Babcock et al., 1987a). BM (NH) 4279 (lectótipo) e BM (NH) 4278 (paralectótipo).

Material examinado. UNG, CuPg-1; GSA/IG-USP, GP/3E4194c.

Discussão. Embora os exemplares descritos por Babcock et al. (1987a) do Devoniano da Bolívia apresentem articulação do tipo gótico curvo, espécimes de $P$. africana da mesma localidade, referidos por Koslowski (1923) e exemplares do Devoniano do Uruguai (Mendéz-Alzola \& Sprechmann, 1973) também mostram articulação do tipo gótico. A presença de nodos e cristas adaperturais em número de 1 a 2 por milímetro ao longo do cordão é outra feição descrita por Babcock et al. (1987a) que não foi observada no material examinado. Curiosamente, essas feições não foram encontradas também em exemplares de P. africana do Devoniano do Uruguai (MendézAlzola \& Sprechmann, 1973) e da Bolívia (Conularia var. striatula Kozloswski, 1923). Entretanto, a preservação de tais feições está intimamente relacionada ao grau de esfoliação da teca (Rodrigues, 2002; Leme, 2002). Adicionalmente, carenas ao longo das arestas (Figura 5.4-5.6) são aqui descritas pela primeira vez para $P$. africana.

É importante notar ainda que Clarke (1913) descreveu $P$. africana para a fauna devoniana da Bacia do Paraná, fundamentado em material proveniente apenas da região de Ponta Grossa. Entretanto, os espécimes referidos por Clarke (1913, estampa VIII, figs. 12-15) como pertencentes a $P$. africana apresentam cordões finos e pouco espaçados. Essas não são feições comuns à $P$. africana. Dessa forma, a única ocorrência registrada de $P$. africana nos estratos da Formação Ponta Grossa é a do material aqui analisado, proveniente da região de Jaguariaíva, representando a primeira ocorrência dessa espécie na bacia do Paraná (vide Leme, 2002).

\section{DISCUSSÃO}

\section{Estruturas internas da teca de conulários}

As feições internas da teca de conulários (septos e carenas) são comumente citadas na discussão da morfologia funcional e afinidades filogenéticas dos conulários (e.g., Kiderlen, 1937; Kozlowski, 1968; Moore \& Harrington, 1956a,b; Bischoff, 1978; Van Iten, 1991a,b; 1992a,b; Jerre, 1994; Van Iten et al., 1996). A morfologia e a microestrutura das feições internas presentes na região das arestas e linhas medianas dos conulários apresentam similaridades em 
número, arranjo, tamanho e forma aos septos gástricos e estruturas da teca dos cifozoários, sendo consideradas estruturas homólogas (Bishoff, 1978; Jerre, 1994; McKinney et al., 1995; Van Iten, 1991a, b; 1992a, b; Van Iten et al., 1996). Dessa maneira, tais estruturas foram interpretadas como estruturas de suporte para os tecidos moles dos conulários, em vida.

Novas ocorrências e informações sobre a anatomia de tecas exibindo estruturas internas fornecem subsídios para um melhor entendimento sobre a variação anatômica e a complexidade morfológica dos Conulatae. Por exemplo, os espécimes de $C$. quichua e $P$. africana aqui descritos apresentam caracteres morfológicos internos da teca preservados. A teca de C. quichua apresenta espessamentos internos na região da linha mediana. Adicionalmente, carenas estão presentes na base e, quando unidas no centro da teca, formam uma estrutura mineralizada, aqui denominada pilar axial (Figura 4.7-4.9). Tal estrutura ainda não foi examinada para os demais gêneros de conulários, constituindo, portanto, estrutura exclusiva de $C$. quichua. Um trabalho de revisão dos gêneros de Conulatae está sendo desenvolvido e poderá contribuir para o maior conhecimento das feições morfológicas (internas e externas) do grupo.

Estrutura similar ao pilar axial, aqui descrito, parece ocorrer na teca de Circonularia eosilurica (Bishoff, 1978). Os septos mineralizados, presentes em C. eosilurica, unemse no centro da cavidade da teca dividindo-a em quatro compartimentos longitudinais (Bishoff, 1978, p. 293, fig. 12). Esse padrão também ocorre em espécies viventes de Stauromedusae (e.g., Craterolophus tethys). Os septos longitudinais de C. tethys não são mineralizados, sendo unidos a um centro comum na região basal. Já na região do cálice os septos convergem para a cavidade gastrovascular (Jerre, 1994). Entretanto, a origem, a função e o significado dessas estruturas na sistemática do grupo não são claras, havendo ainda a necessidade de exame de maior número de exemplares com tais feições preservadas. Do mesmo modo, a origem e a função das pontuações irregularmente distribuídas na região da abertura (Figura 4.13, 4.14) não é conhecida.

Assim como C. quichua, a outra espécie estudada, isto é, P. africana, apresenta carenas ao longo das arestas (Figura 5.4-5.6). Outras espécies do mesmo gênero exibem estrutura similar. Por exemplo, P. crustula, do Carbonífero da América do Norte apresenta espessamentos internos nas arestas, diferindo de $P$. africana por apresentar também septos (Van Iten, 1992a). Finalmente, os dados acima mostram que a morfologia interna de $C$. quichua e $P$. africana é mais complexa do que aquela descrita por Clarke (1913) e por Babcock et al. (1987a).

\section{Significado paleoecológico e paleobiogeográfico da fauna de conulários da Bacia do Paraná}

Clarke (1913) identificou as espécies $P$. africana, $P$. ulrichana e $C$. quichua nos sedimentitos da Formação Ponta Grossa, nas regiões de Tibagi, Jaguariaíva e Ponta Grossa (PR). De acordo com a revisão, P. ulrichana (Clarke, 1913) não foi encontrada no material estudado, o que não significa, obrigatoriamente, que não esteja presente na Formação Ponta Grossa. Adicionalmente, a fauna de conulários da Formação Ponta Grossa (sub-bacia Apucarana) pertence a gêneros e espécies cosmopolitas, sendo amplamente dominada por C. quichua e pouco diversificada, especialmente quando comparada com a fauna coeva da Bolívia, caracterizada por seis espécies (C. quichua, C. albertensis, $P$. africana, $P$. ulrichana e Reticulaconularia baini, Malvinoconularia cahuanotensis).

Considerando as paleolatitudes do intervalo de ocorrência dos conulários da Bolívia e da bacia do Paraná, a sua distribuição latitudinal é apreciável. Durante o Devoniano, os conulários bolivianos viveram em águas situadas em torno dos $45^{\circ}$ e $50^{\circ}$ de latitude sul e os da bacia do Paraná, próximas aos $70^{\circ}$ de latitude sul. Portanto, as espécies que colonizaram a bacia do Paraná estavam submetidas a condições ambientais de águas muito frias, em decorrência da proximidade do pólo, na época. As espécies $C$. quichua e $P$. africana devem ter sido, portanto, euritérmicas.

A ampla distribuição de algumas espécies de conulários no Domínio Malvinocáfrico (Richter \& Richter, 1942) sugere também que sua ontogênese deu-se a partir de larvas planctônicas, teleplânicas (sensu Scheltelma, 1989), isso é, com capacidade de sobreviver nas correntes oceânicas por prolongados períodos de tempo. Dados disponíveis para invertebrados marinhos viventes sugerem, por exemplo, que as larvas teleplânicas têm dispersão transoceânica, sendo encontradas tanto no oceano Atlântico como no Pacífico (Ventura \& Pires, 2002).

\section{CONCLUSÕES}

Conulários, representados pelas espécies cosmopolitas C. quichua e $P$. africana, são macrofósseis comuns no registro fóssil da Formação Ponta Grossa, no Estado do Paraná. São particularmente abundantes nos sedimentitos que afloram na seção geológica do município de Jaguariaíva. A fauna de conulários é dominada por C. quichua, sendo rara a ocorrência de $P$. africana, espécie aqui registrada pela primeira vez para a região de Jaguariaíva. A fauna de conulários da Formação Ponta Grossa do Paraná é, portanto, pouco diversificada, especialmente se comparada com faunas coevas da Bolívia. Finalmente, a morfologia de $C$. quichua e $P$. africa$n a$, como aqui descrita, é mais complexa do que a descrita por Clarke (1913) e Babcock et al. (1987a).

\section{AGRADECIMENTOS}

O presente estudo contou com o auxílio dos pesquisadores A.C. Marques (IB/USP), N. Bernardi (IBB/UNESP), S. Petri (IG/USP), M.J. Garcia (UNG), R.C.T. Cassab (CPRM), L.E. Anelli (IG/USP), M.L.N. Fransozo (IBB/UNESP) e E. Bosetti (UEPG). Os autores agradecem também aos alunos do GSA/IG-USP, R.P. Ghilardi, A.M.F. Sales, L.H.C. Mello, F.F. Torello, além de H.A. Rodrigues (IBB/UNESP) pelo auxílio em campo, e a L.B. Simões e L.E. Anelli pelo trabalho 
fotográfico. Aos assessores da RBP, pelas sugestões ao manuscrito. Suporte financeiro foi oferecido pela FAPESP, pelo CNPq e pelo Hanover College Faculty Development Committee (Hanover, USA).

\section{REFERÊNCIAS}

Ahfeld, F. \& Branisa, L. 1960. Geología de Bolívia. $1^{\text {a }}$ ed. La Paz, Instituto Boliviano del Petroleo, 245 p.

Azevedo-Soares, H.L.C. 1999. Classe Tentaculita na Formação Ponta Grossa: uma proposta de sistematização. Programa de Pós-Graduação em Geologia, Universidade Federal do Rio de Janeiro, Dissertação de Mestrado, 132 p.

Babcock, L.E. 1991. The enigma of conulariid affinities. In: A.M. Simonetta \& S.C. Morris (eds.) The early evolution of Metazoa and the significance of problematic fossil taxa, Cambridge University Press, p. 113-143.

Babcock, L.E. \& Feldmann, R.M. 1986. Devonian and Mississipian conulariids of North America. Part A. General discription and Conularia. Annals of Carnegie Museum, 55:349-410.

Babcock, L.E.; Feldmann, R.M.; Wilson, M.T. \& Suárez-Riglos, M. 1987a. Devonian conulariids of Bolivia. National Geographic Research, 3:210-231.

Babcock, L.E.; Feldmann, R.M. \& Wilson, M.T. 1987b. Teratology and pathology of some Paleozoic conulariids. Lethaia, 20:93105.

Babcock, L.E.; Gray, J.; Boucot, A.J.; Himes, G.T. \& Siegele, P.K. 1990. First Silurian conulariids from Paraguay. Journal of Paleontology, 64(6):897-902.

Bergamaschi, S. 1999. Análise estratigráfica do Siluro-Devoniano (Formação Furnas e Ponta Grossa) da sub-bacia de Apucarana, bacia do Paraná, Brasil. Programa de Pós-graduação em Geologia Sedimentar, Universidade de São Paulo, Tese de Doutorado, $167 \mathrm{p}$.

Bergamaschi, S. \& Pereira, E. 2001. Caracterização de seqüências deposicionais de $3^{\mathrm{a}}$ ordem para o Siluro-Devoniano na subbacia de Apucarana, bacia do Paraná, Brasil. In: J.H.G. Melo \& G.J.S. Terra (eds.) Correlações de Seqüências Paleozóicas SulAmericanas, Ciência Técnica Petróleo, Seção Exploração de Petróleo, 20:63.

Bernard, F. 1895. Élements de Paléontologie. $1^{\text {a }}$ ed. Paris, J. Libraire, B. Bailliere \& Fils, 1166 p.

Bischoff, G.C.O. 1978. Internal structures of conulariid tests and Circonulariina n. suborder (Cnidaria, Scyphozoa). Senckenbergiana Lethaea, 59:275-327.

Bosetti, E.P. 1989. Revisão sistemática dos Lingulida (Brachiopoda: Inarticulata) da Formação Ponta Grossa (Devoniano), Estado do Paraná, Brasil. In: CONGRESSO BRASILEIRO DE PALEONTOLOGIA, 11, 1989. Anais , Curitiba, SBP, p. 73.

Boucek, B. 1939. Conularida. In: O.H. Schindewolf (ed.) Handbuch der Palaeozoologie, Gerbruder Borntraeger, p. 111131.

Branisa, L. 1965. Los fosiles guias de Bolivia. Servicio Geologico de Bolivia, p. 282 (Boletín 6).

Ciguel, J.H.G. 1989. Bioestratigrafia dos Tentaculoidea no flanco oriental da Bacia do Paraná e sua ocorrência na América do Sul (Ordoviciano-Devoniano). Programa de Pós-graduação em Geologia Sedimentar, Universidade de São Paulo, Dissertação de Mestrado, $237 \mathrm{p}$.

Clarke, J.M. 1913. Fósseis devonianos do Paraná. Serviço Geológico e Mineralógico do Brasil, 353 p. (Monografia 1).
Collins, A.G.; Marques A.C. \& Simões, M.G. 2000. The phylogenetic placement of Conulatae within Cnidaria. Geological Society of America, Abstracts with Programs, 32(7):A-443.

Douglas, J.A. 1920. Geological sections through the Andes of Peru and Bolivia: II. From the port of Mollendo to the Inambari River. Quaternaly Journal of the Geological Society of London, 76(301):1-61.

Feldmann, R.M.; Chapman, R.E. \& Hannibal, J.T. 1989. Paleotechiniques. The Paleontological Society, 358 p. (Special Publication 4).

Grahn, Y.; Pereira, E. \& Bergamaschi, S. 2000. Silurian and Lower Devonian chitinozoan biostratigraphy of the Paraná Basin in Brazil and Paraguay. Palynology, 24:147-176.

Hansman, R.H.; Shaw, F.C. \& Pettyjohn, W.A. 1962. Supplement to the catalog of the type specimens of fossils in the University of Cincinnati Museum. $1^{\text {a }}$ ed. Cincinnati, University of Cincinnati Press, $131 \mathrm{p}$.

Ivantsov A.Y. \& Fedonkin M.A. 2002. Conulariid-like fossil from the Vendian of Russia: a metazoan clade across the Proterozoic/ Palaeozoic boundary. Palaeontology, 45:1219-1229.

Jerre, F. 1993. Conulariid microfossils from the silurian Lower Visby Beds of Gotland, Sweden. Paleontology, 36:403-424.

Jerre, F. 1994. Anatomy and phylogenetic significance of Eoconularia loculata, a conulariid from the Silurian of Gotland. Lethaia, 27:97-109.

Kayser, F.H.E. 1897. Beiträge zur Kenntniss einiger paläozischer Faunen Sud-Amerikas. Deutsche geologische Gesellschaft, Zeitschrift, 49(2):274-317.

Kiderlen, H. 1937. Die Conularien. Über Bau and Leben der ersten Scyphozoa. Neues Jahrbuch für Mineralogie, Beilage- Band, 77:113-169.

Kotzian, C.B. 1995. Estudo sistemático e morfo-funcional de bivalves (Mollusca) das formações Vila Maria (Siluriano) $e$ Ponta Grossa (Devoniano), bacia do Paraná, Brasil: interpretação do regime hidrodinâmico-sedimentar. Programa de Pósgraduação em Geociências, Universidade Federal de Rio Grande do Sul, Tese de Doutorado, 378 p.

Kozlowski, R. 1913. Fossiles Devoniens de l'État de Paraná (Brésil). Annales de Paleontologie, 8(1/2):14-19.

Kozlowski, R. 1923. Fauna Devonienne de Bolivie. Annales de Paleontologie, 12(1/2):1-112.

Kozlowski, R. 1968. Nouvelles observations sur les conulaires. Acta Palaeontologica Polonica, 13:497-535.

Lange, F.W. \& Petri, S. 1967. The Devonian of the Paraná Basin. In: J.J. Bigarella (ed.) Problems in Brazilian Devonian Geology, Boletim Paranaense de Geociências, 21/22:5-55.

Leme, J.M. 2002. Revisão sistemática dos Conulatae Collins et al. 2000, Formação Ponta Grossa, Devoniano (?LochkovianoFrasniano), Bacia do Paraná, Brasil: implicações paleobiogeográficas e comentários sobre as relações filogenéticas entre os Conulatae. Programa de Pós-graduação em Geologia Sedimentar, Universidade de São Paulo, Dissertação de Mestrado, 100 p.

Lof, P. 1985. Elsevier's Invertebrate Fossils Chart. Elsevier, Amsterdam, $156 \mathrm{p}$.

Machado, D.M.C. 1999. Nuculites Conrad, 1841 (Mollusca, Bivalvia): sistemática e implicações paleobiogeográfica. Programa de Pós-graduação em Geociências, Universidade Federal do Rio Grande do Sul, Tese de Doutorado, 298 p.

McKinney, F.K.; Devolvé, J.J. \& Sobieraj, J. 1995. Conularia sp. from the Pyrénées: further support for scyphozoan affinities of the Conularida. Lethaia, 28:229-236. 
McMenamin, M.A.S. 1987. The fate of Ediacaran Fauna, the nature of conulariids, and the basal paleozoic predator revolution. Geological Society of America, Abstracts with Programs, 19:29.

Mendéz-Alzola, R. \& Sprechmann, P.G. 1973. Fauna del Devonico Temprano del Uruguay, II. Sobre representantes de Conularia y Mesoconularia (Conulariidae, Conulariinae). Revista de Biologia del Uruguay, 1(2):129-138.

Moore, R.C. \& Harrington, H.J. 1956a. Scyphozoa. In: R.C. Moore (ed.) Treatise on Invertebrate Paleontology, Part F, Coelenterata, Geological Society of America and University of Kansas Press, F27-F38.

Moore, R.C. \& Harrington, H.J. 1956b. Conulata. In: R.C. Moore (ed.) Treatise on Invertebrate Paleontology, Part F, Coelenterata, Geological Society of America and University of Kansas Press, F54-F66.

Petri, S. 1948. Contribuição ao estudo do Devoniano paranaense. Boletim do Departamento Nacional da Produção Mineral, 129:1-125.

Petri, S. \& Fúlfaro, V.J. 1983. Geologia do Brasil: Fanerozóico. $1^{\mathrm{a}}$ ed. São Paulo, EDUSP, 631 p.

Popp, M.T.B. 1985. Revisão dos trilobitas calmoniídeos e comunidades faunísticas da Formação Ponta Grossa, Devoniano do Estado do Paraná. Programa de Pós-graduação em Geociências, Universidade Federal do Rio Grande do Sul, Tese de Doutorado, $112 \mathrm{p}$.

Quadros, R. 1987. Paleontologia dos Brachiopoda - Lingulida, Strophomenida, Spiriferina, Terebratulida - devonianos da Serra de Atimã e arredores, Mato Grosso, Brasil. Programa de Pós-graduação em Geociências, Universidade Federal do Rio Grande do Sul, Tese de Doutorado, $87 \mathrm{p}$.

Reed, F.R.C. 1904. Mollusca from the Bokkeveld Beds. South African Museum Annals, 4:239-274.

Reed, F.R.C. 1925. Revision of the fauna of the Bokkeveld Beds. South African Museum Annals, 22:27-225.

Richter, R. \& Richter, E. 1942. Die Trilobiten der Weismer-Schichten am Hohen Venn, mit Bemerkunger über die Malvinocaffrisch Provinz. Senckenbergische Naturforscher, Gesellschafte, Ablandlungen 25:156-179.

Rodrigues, S.C. 2002. Tafonomia comparada dos Conulatae Collins, et al. 2000, Formação Ponta Grossa, Devoniano (?Lochkoviano-Frasniano), bacia do Paraná: implicações paleoeautocológicas e paleoambientais. Programa de Pós-graduação em Geologia Sedimentar, Universidade de São Paulo, Dissertação de Mestrado, $100 \mathrm{p}$.

Scheltelma, R.S. 1989. Planktonic and non-planktonic development among prosobranch gastropods and its relationship to the geographic range of species. In: J.S. Ryland \& P.A. Tyler (eds.) Reproduction, genetics and distributions of marine organisms. European Marine Biology Symposia, 23. Olsen \& Olsen, Fredensborg, p. 183-188.

Schwarz, E.H.L. 1906. South African Palaeozoic fossils. Albany Museum, Records, 1:347-404.

Sharpe, D. 1856. Descriptions of Palaeozoic Mollusca from South Africa. Transactions of the Geological Society of London, 2(7):206-215.
Simões, M.G.; Ghilardi, R.P.; Sales, A.M.F.; Rodrigues,S.C. \& Leme, J.M. 2000a. Taphonomy and genesis of the "Conularia Beds" from Ponta Grossa Formation (Devonian) within a sequence stratigraphy framework. In: PALEO 2000. Resumos, Botucatu, Núcleo SBP/SP, p. 20.

Simões, M.G.; Mello, L.H.C.; Rodrigues, S.C; Leme, J.M. \& Marques, A.C. 2000b. Conulariid taphonomy as a tool in paleoenvironmental analysis. Revista Brasileira de Geociências, 30(4): 757-762.

Sinclair, G.W. 1940. A discussion of the genus Metaconularia with descriptions of new species. Transactions of the Royal Society of Canada, 34:101-121.

Sinclair, G.W. 1942. The Chazy Conularida and their congeners. Annals of the Carnegie Museum, 29:219-240.

Sinclair, G.W. 1948. The biology of the Conularia. McGill University, Ph.D. Thesis, $442 \mathrm{p}$.

Sinclair, G.W. 1952. A classification of the Conularida. Fieldiana Geology, 10(13):135-145.

Siviero, F.N. 2002. Revisão sistemática das conulárias brasileiras. Programa de Pós-graduação em Geologia, Universidade Federal do Rio de Janeiro, Dissertação de Mestrado, 80 p.

Sowerby, J. 1820 (datado 1821). The mineral conchology of Great Britain; or coloured figures and descriptions of those remains of testaceous animals or shells, which have been preserved at variouus times and depths in the Earth, 3, Parte 46. Londres, $194 \mathrm{p}$.

Steinmann, G. 1930. Geologie von Perú. Carl Winters Universitätsbuchhndlung, Heidelberg, $448 \mathrm{p}$.

Steinmann, G. \& Doderlein L. 1890. Elemente der Paläontologie. Verlag von Wilhelm Engelmann, Leipzig, 848 p.

Thomas, I. 1905. Neue Beiträge zur Kenntnis der devonischen Fauna Argentiniens. Deutsche Geologische Gesellschft, Zeitschrift, 57:233-290.

Ulrich, A. 1892. Palaeozoische Versteinerungen aus Bolivien. Neues Jahrbuch für Mineralogie, Geologie und Paläontologie, 8:5-116.

Van Iten, H. 1991a. Evolutionary affinities of conulariids. In: A.M. Simonetta \& S.C. Morris (eds.) The early evolution of Metazoa and the significance of problematic fossil taxa, Cambridge University Press, p. 145-155.

Van Iten, H. 1991b. Anatomy, patterns of occurrence, and nature of the conulariid schott. Palaeontology, 34:939-954.

Van Iten, H. 1992a. Morphology and phylogenetic significance of the corners and midlines of the conulariid test. Paleontology, 35:335-358.

Van Iten, H. 1992b. Microstructure and growth of the conulariid test: implications for conulariid affinities. Paleontology, 35:359-372.

Van Iten, H.; Fitzke, J.A. \& Cox, R.S. 1996. Problematical fossil cnidarians from the Upper Ordovician of the North-Central USA. Palaeontology, 39:1037-1064.

Ventura, C.R.R. \& Pires, D.O. 2002. Ciclo de vida de invertebrados marinhos. In: C.R.R. Ventura \& D.O. Pires (eds.) Biologia Marinha, Interciência, p. 50-67.

Walcott, C.D. 1886. Second contribution to the studies on the Cambrian faunas of North America. Bulletin of the United States Geological Survey, 30:1-369. 